\title{
Determination of THE CURRENT STATE OF Human RETINA USING Algorithms OF ClASSIFICATION
}

\author{
Olga Titova, Dmitry Anisimov, Oleg Kolosov
}
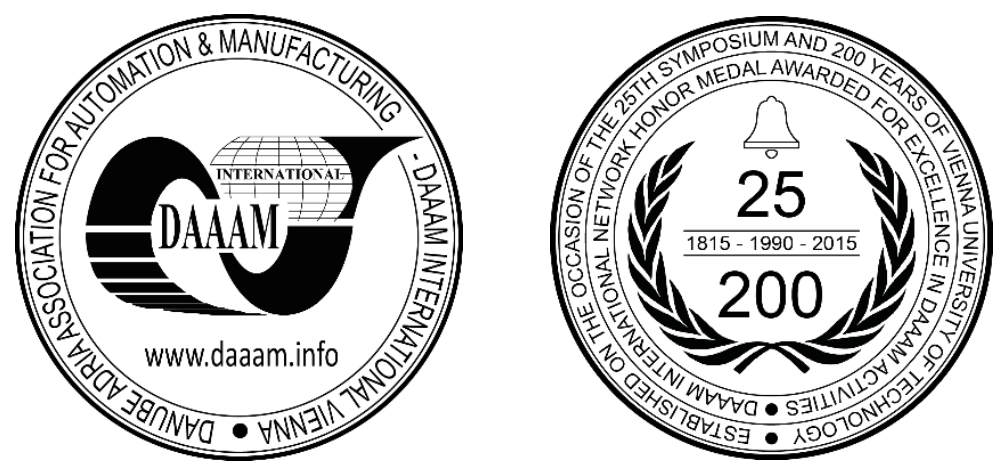

This Publication has to be referred as: Titova, O[lga]; Anisimov, D[mitry] \& Kolosov, O[leg] (2018). Determination of the Current State of Human Retina Using Algorithms of Classification, Proceedings of the 29th DAAAM International Symposium, pp.1209-1215, B. Katalinic (Ed.), Published by DAAAM International, ISBN 978-3-902734-20-4, ISSN 1726-9679, Vienna, Austria

DOI: $10.2507 / 29$ th.daaam.proceedings. 175

\begin{abstract}
The ERG (electroretinogram) is a diagnostic test that measures the electrical activity generated by neural and nonneuronal cells in the retina in response to a light stimulus [1], [2]. This test is used worldwide to assess the status of the retina in eye diseases in human patients. In the present study, we aim to develop algorithm for determining the current state of the human retina based on the ERG. We analyse standard and rhythmic ERG in the time and frequency domain and use these characteristics for feature extraction and classification. By comparing results obtained using various methods of dimensional reducing and classification we achieved a sufficiently high quality classification of selected pathologies.
\end{abstract}

Keywords: electroretinogram; feature extraction; dimensional reduction; classification

\section{Introduction}

With the development of science and technology, it became possible to automate many complicated processes, including the assignment of objects to a particular group based on formal features, or classification. At present, classification methods are used to solve a variety of diverse and non-trivial scientific and practical problems [3].

Among other things, classification methods are widely used in solving problems of automated medical diagnostics, characterized by a large number of diagnostic features and a high level of intersection of feature spaces for various states of the object of study. Professionalism of medical staff plays the decisive role in the correctness of diagnosis, often the processing and analysis of the entire spectrum of research takes a large amount of time. In order to speed up the process of analysing information and improve the quality of the decisions made, automating of collection, analysis of information and algorithmization of decision-making based on some formal features can be applied.

Development and implementation of intelligent medical diagnosis systems in medical organizations will reduce the risk of errors in diagnosis, use hidden features and dependencies in diagnostics, and automate some time-consuming information analysis processes. The question of automatic determination of the state of various systems of the human body can often been found in scientific papers. For example, there are investigations about the automatic detection of vocal cord/vocal tract diseases from the medically disordered speech [4], or about automatic detection of selected pronunciation pathology in preschool children [5]. 
In Saratov State Technical University, when forming the method of automated diagnosis of diseases of the urinary system, a neural network such as a radial basis function was used [6], in Povolzhsky Institute Of Management, together with the vision correction center has been developed a system for diagnosing glaucoma stages using the fundus image based on Kohonen's self-organizing networks [7]. In scientific papers from VSTU, approaches to automated diagnosis of nephropathy [8] and the solution of the problem of rapid diagnosis of primary forms of blood supply to the brain [9], using discriminant analysis and other methods [10], [11] are described.

This study is devoted to the task of automated medical diagnostics, that can be formulated as determination the pathologies of the human retina based on the complex of electrophysiological studies - electroretinograms (ERG). In other words, we need to solve the problem of automated medical diagnostics of the current state of a complex biological object with intersecting areas of features using the example of determining the current state of the retina based on a complex of electroretinographic studies.

\section{Method}

The classification task described above is one of the types of data mining tasks or KDD (knowledge discovery in database) [12]. The solution of this type of problems in general contains several successive steps, including data preprocessing and verification of results. Below is a list of the steps:

1. Data selection.

2. Data preparation.

3. Transformation of data.

4. Data mining.

5. Interpretation.

\subsection{Data selection}

As the initial data, we used ERG, produced by specialists of the Institute of Eye Diseases after Helmholtz in Moscow, Russia for the period from 2009 to 2012. Registration of ERG has been implemented on the electrophysiological system TOMEY EP-1000 (Japan).

In ophthalmology, an electrophysiological method called "electroretinography" is used to assess the state of the visual analyser and early diagnose pathological changes in cells. An electroretinogram (ERG) occurs when light stimuli (testing input signals) are applied to the retina of various sizes, shapes, wavelengths, intensities, durations, repetition rates under different light and dark adaptation conditions. The practical value of electroretinography is determined by the fact that it is a very sensitive method for assessing the functional state of the retina. In studies of the retina, a whole complex of signals is used: standard ERG, rhythmic ERG, pattern-ERG, evoked potentials and others [1], [2].

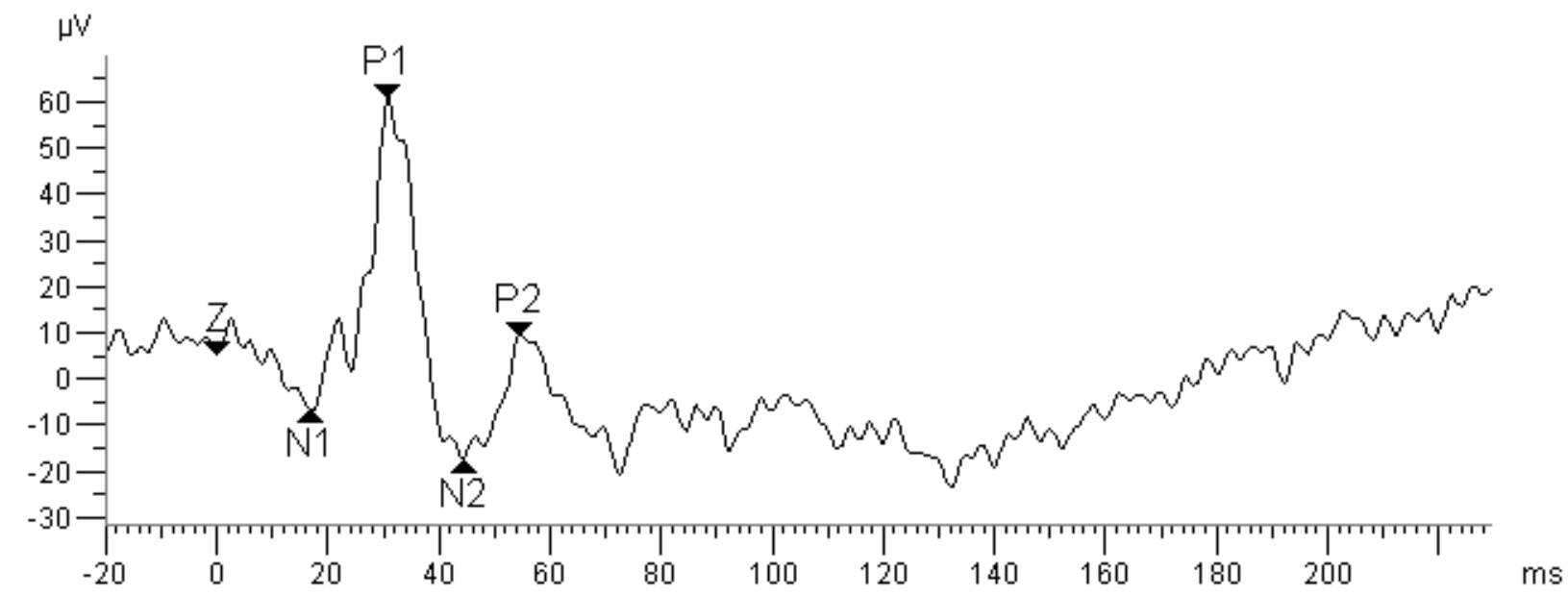

Fig. 1. Standard ERG, received by the the electrophysiological system TOMEY EP-1000 (Japan).

To analyse the current state of the retina, this work examines a set of electroretinograms: standard ERGs that reflect the biological potential of the retina in response to the single light flash, and photopic rhythmic ERGs that reflect the biological potential of the retina in response to a testing effect as a series of periodic flashes with a certain frequency. The flash rates are standard: $8.3 \mathrm{~Hz}, 10 \mathrm{~Hz}, 12 \mathrm{~Hz}, 24 \mathrm{~Hz}$ and $30 \mathrm{~Hz}$. Five pathologies are investigated in this study: glaucoma (GL), chromosomal retinoschisis (XR), diabetic retinopathy (DR), central serous chorioretinopathy (CSH), multiple sclerosis (NEU). 


\subsection{Data preparation (feature extraction and data cleansing )}

To obtain the initial features for the classification of standard ERG signals, it is necessary to move from the description of an object in the form of signals to a characteristic description in the form of formal real signs. Specialists for the study of ERG and diagnosis use the numerical characteristics of the ERG, indicated in Fig. 1. From the point of view of experts, the abscissas and ordinates of the first two extrema of the signal have the greatest significance for diagnostics.

In previous studies on this topic [13], it was proved that the initial features extracted from the standard ERG are not enough to qualitatively classify the current state of the retina and it is necessary to include additional informative features in the classifier, for example, various non-linear combinations of characteristic signal points.

In addition, to improve the quality of classification, it is necessary to analyze the complex of ERG, which includes various signals. For the current study, we chose a rhythmic ERG. To extract features from rhythmic ERGs, we turn to frequency characteristics. The spectral characteristics are obtained from the amplitudes of the harmonics in the decomposition of signals in a Fourier series, according to formulas (1), (2).

$$
f(x)=\frac{a_{0}}{2}+\sum_{n=1}^{N-1} A_{n} \cos \left(\frac{2 \pi n x}{T}+\theta_{n}\right)
$$

the coefficients of this equation can be determined as follows:

$$
\left\{\begin{array}{c}
a_{n}=\frac{2 \Delta t}{T} \sum_{i=0}^{N-1} X_{i} \cdot \cos \left(\frac{1}{T} \cdot 2 \pi \cdot \Delta t \cdot i\right) \\
b_{n}=\frac{2 \Delta t}{T} \sum_{i=0}^{N-1} X_{i} \cdot \sin \left(\frac{1}{T} \cdot 2 \pi \cdot \Delta t \cdot i\right) \\
A_{n}=\sqrt{{a_{n}{ }^{2}+b_{n}{ }^{2}}_{\theta_{n}}}=\operatorname{arctg} \frac{b_{n}}{a_{n}}
\end{array}\right.
$$

The frequency characteristic of the object can be obtained as an attitude of the spectrum of the output signal to the spectrum of the input signal. The process of transformation of the input test signal in the rERG can be represented by the functional diagram shown in Fig. 2

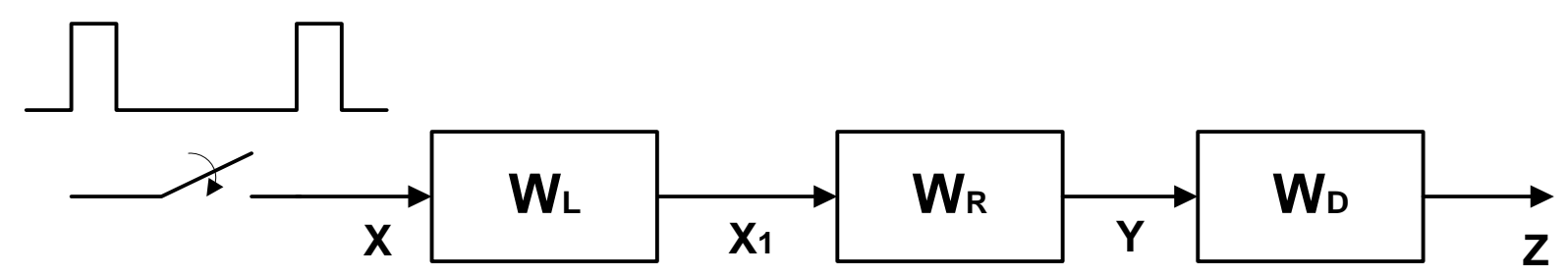

Fig. 2. Functional diagram of rERG $(\mathrm{Z}(\mathrm{x}))$ signal acquisition

Thus, the spectrum of the recorded signal rERG Z(f) represents the spectrum of the input signal X(f) converted by three dynamic links (lamp-flash $\left(\mathrm{W}_{\mathrm{L}}\right)$, retina $\left(\mathrm{W}_{\mathrm{R}}\right)$ and recording instrument $\left(\mathrm{W}_{\mathrm{D}}\right)$ ). Such a process can be written as follows:

$$
Z(f)=X(f) \frac{X_{1}(f)}{X(f)} \cdot \frac{Y(f)}{X_{1}(f)} \cdot \frac{Z(f)}{Y(f)}=X(f) \cdot W_{L}(f) \cdot W_{R}(f) \cdot W_{D}(f)
$$

To obtain directly the frequency response of the retina, we need to take into account the frequency properties of the flash lamp and recording instrument. The final characteristic is presented in Fig. 3. 


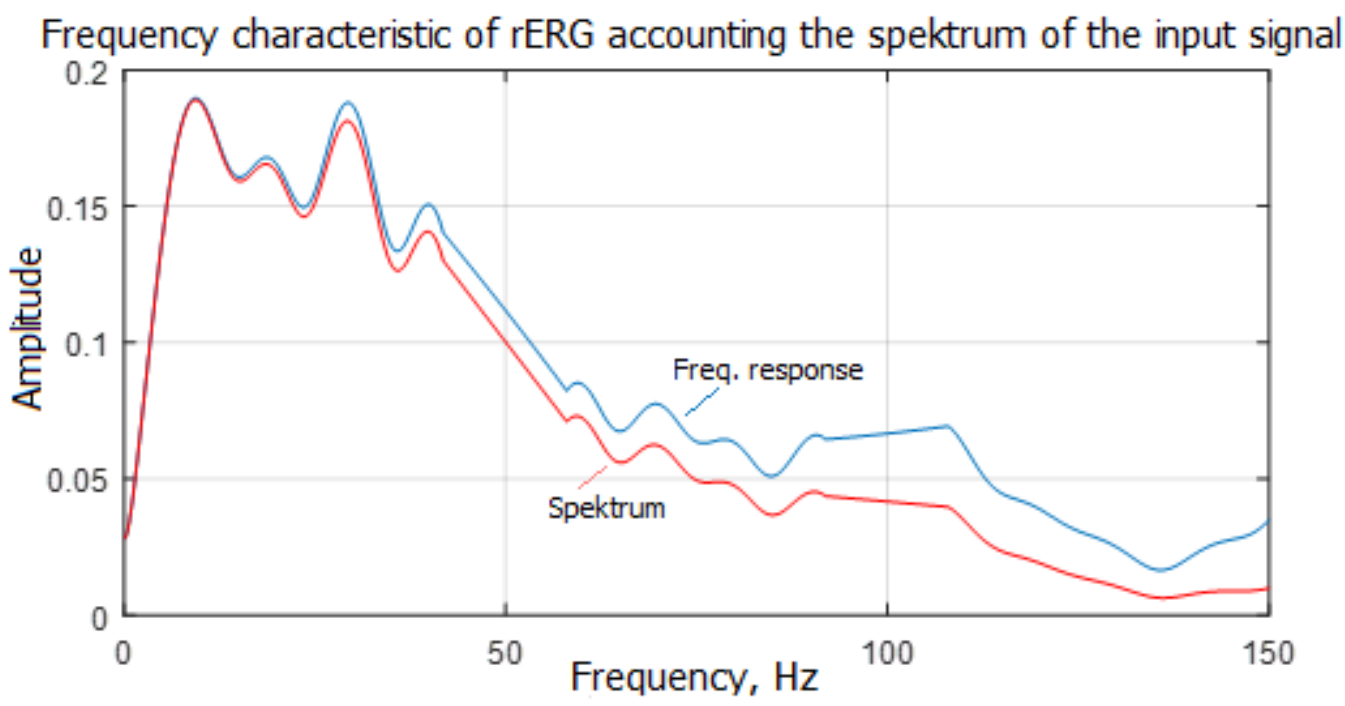

Fig. 3. Frequency response of the rERG

In order to use the obtained information about the frequency properties of the retina as input data of the classifier, it is necessary to proceed to the description in the form of numerical parameters. In the current study for this purpose it is proposed to use the coefficients of polynomials approximating the frequency function.

Investigating the type of retardation frequency response, we can distinguish two stable sections where the behaviour of the function can be described by simple polynomials. In the section from 0 to $50 \mathrm{~Hz}$, the approximating function has the form of a second degree polynomial, and in the section from $50 \mathrm{~Hz}$ - a linear function. Equations of polynomials are:

$$
g_{1}=\operatorname{argmin} \sum_{i=1}^{N}\left(y_{i}-\left(b_{0}+b_{1} x_{i}+b_{2} x_{i}^{2}\right)\right)^{2} ; g_{2}=\operatorname{argmin} \sum_{i=1}^{N}\left(y_{i}-\left(a_{0}+a_{1} x_{i}\right)\right)^{2}
$$

Thus, we obtained the description of the retina in the form of a matrix object-feature. The original number of features is 34. Further, the obtained description of the object in the form of a matrix of signs is clearing of abnormal observations, each sign is checking for a normal distribution and the values are normalizing for further application of methods for dimensional reducing and classification.

\subsection{Data transformation (dimensional reduction)}

There are two types of dimensional reducing methods:

- $\quad$ selection of informative features (feature selection);

- synthesis of informative features (feature extraction).

For the study, we take the methods of each type and compare the influence of the reduction method on the classification results. As a method for the synthesis of informative features, we will use the principal component method (PCA), and as a selection method, we will use the methods of greedy search for inclusion (Forward Selection) and exclusion (Backward Elimination). Such a choice is due to the speed of the methods and the relatively high quality of selection in most cases.

\subsection{Data mining (classification)}

In this study, we use four classification algorithms and investigate the quality of classification depending on the set of input parameters of the classifier [14]. The algorithms investigated are listed below:

- naive Bayes classifier

- linear discriminant analysis

- support vector machine

- neural network with one hidden layer and sigmoid activation function

These methods were chosen for the study due to low computational complexity and high learning speed with a fairly good quality of classification. For each classification method, we analyze the dependence of the quality of classification on the choice of the method of reducing the feature space and its parameters. Under the parameters of the reduction method must be understood the number of attributes in the training sample after reducing the dimension. The maximum number of parameters for each method of reducing the dimension is determined by the criterion of significance $\alpha=0,05$. 


\subsection{Interpretation}

Assessment of the quality of the classifier is carried out by the method of cross-validation (cross-checking). The entire initial sample is divided randomly into 10 subsamples of the same size and 10 circles of training and verification are conducted, with 9 random subsamples acting as a training sample on each circle, and the tenth as a test one. As a result, quality indicators are obtained in the form of percentages correctly assigned to each class of objects.

\section{Results}

In the course of conducting research based on the criterion for minimizing errors, optimal sets of attributes were chosen for each method of reducing the dimension and the classification method. By an optimal set of features, we mean a set that allows to achieve the maximum quality of classification. Fig. 4 presents the classification results for each method using the optimal set of features. This figure shows that, regardless of the classification method chosen, the principal component method (PCA) makes it possible to achieve the best classification quality.

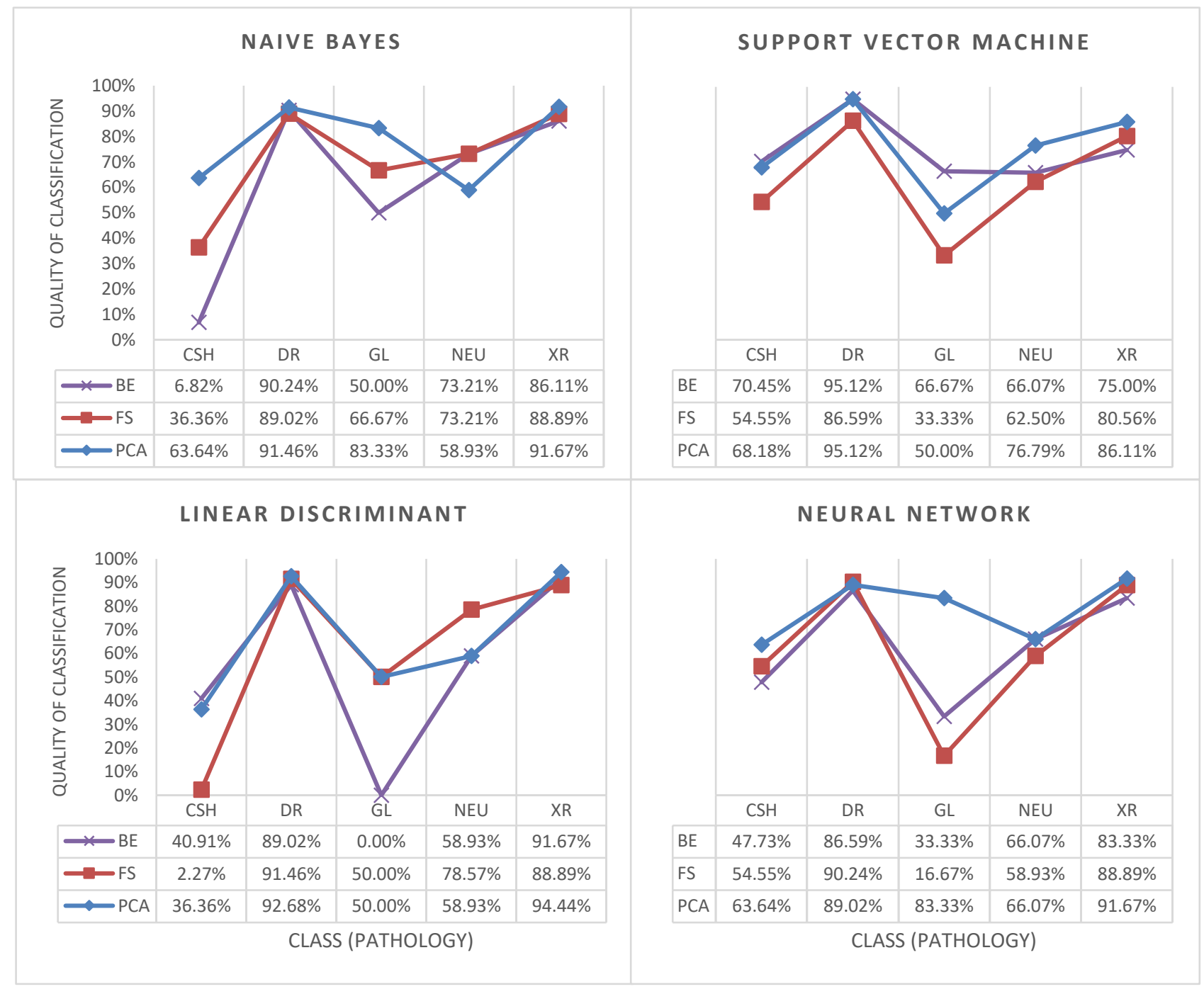

Fig. 4. Comparison of classification results for each dimensionality reduction method using the optimal set of parameters

In order to evaluate the improvement in the quality of classification, after including additional features from the frequency characteristics, we will conduct a comparative analysis of the quality of classification using the following three sets of parameters:

1. The initial set of features that includes only characteristic points of the standard ERG.

2. A complete set of features, including the characteristic points of the ERG, their combinations and all the features extracted from the rERG.

3. The optimal set of features, including only features providing the best classification quality among all reduction methods and feature sets. 
The quality of classification when training classifiers on the initial set is below the threshold value. Glaucoma (GL) shows the lowest quality indicators, the part of correctly defined measurements does not exceed $33 \%$, also central serous chorioretinopathy (CSH) has poor quality of the classification. Training classifiers on the full set of features gives a similar result. Graphically, the results of the comparison are presented on Fig. 5.

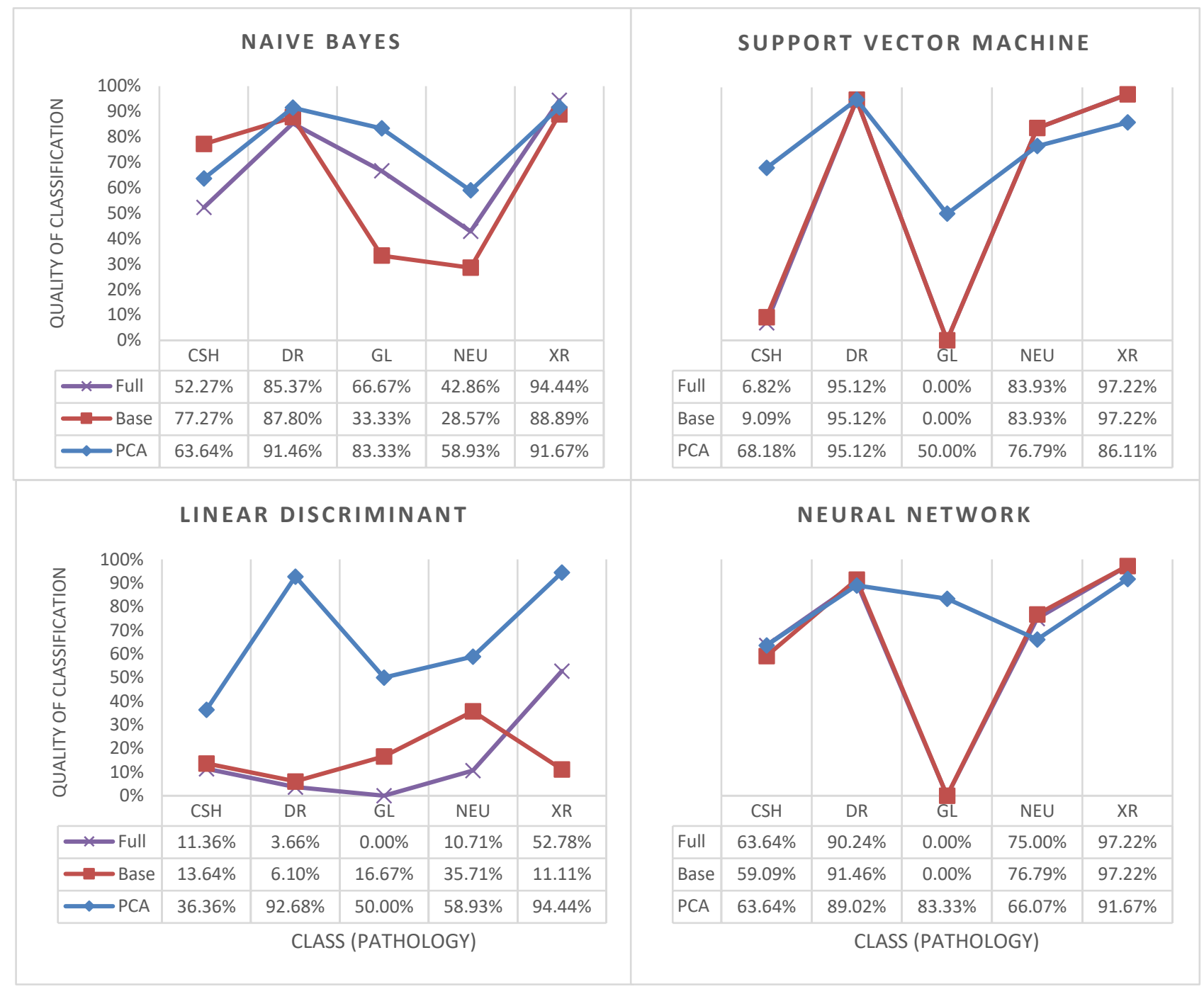

Fig. 5. Comparison of classification results using optimal, full and base set of parameters

The figure show that the lines corresponding to the quality indicators in cases of use optimal feature sets, are on average higher than the lines corresponding to the initial and full set. Based on these results, it can be argued that the use of additional diagnostic features without analyzing and selecting informative ones does not improve the result. The implementation of dimensional reducing methods to the object-to-sign matrix eliminates contradictions in the values of features and significantly improves the quality of classification for glaucoma, central serous chorioretinopathy (CSH), while maintaining the quality of classification in other pathologies.

\section{Conclusion}

In this study, we set ourselves two main tasks: extracting useful information from ERG signals for use as classifier parameters and solving the problem of determining the current state of the retina based on these parameters with a satisfactory classification quality. To solve the first problem, we investigated standard and rhythmic ERG in the time and frequency domain, obtained the frequency response of the retina as a dynamic object, approximated this characteristic, and obtained a set of diagnostic features. Next, we investigated different methods of reducing the dimension and classification, conducted a comparative analysis of the quality of classification for all possible combinations of methods and sets of parameters.

The results obtained in this study meet the accuracy requirements for the tasks of automated medical diagnostics; the average quality rating of the classification exceeds $75 \%$. This shows the high potential of ERGs in order to build systems for the automatic diagnosis of the state of the retina. 
Further development of this study may be an increase in the number of diagnosed pathologies and an improvement in the quality of classification. In order to further improve the diagnostic quality, it is necessary to use complicated systems with a parallel structure, which can reduce the degree of intersection of diagnostic features for different states of an object. An example of such a system could be a system of binary classifiers providing the highest quality in determination of individual pathologies with further aggregation of the results. Another possible way to improve the quality may be an addition of new diagnostic features from other types of ERG to the description of the object. For example, pattern-ERG (PERG) - the retinal response to light exposure in the form of a chessboard.

\section{Acknowledgments}

This paper was prepared with the financial support of Russian Foundation for Basic Research (Project № 16-01-00054a). The authors gratefully acknowledge the support of the Faculty of Automation and Computer Engineering of the Moscow Power Engineering Institute and the Center for Eye Diseases after Helmholtz for this work.

\section{References}

[1] M. F. Marmor, A. B. Fulton, G. E. Holder, Y. Miyake, M. Brigell and M. Bach, "ISCEV Standard for full-field clinical electroretinography (2008 update)" (2009). Doc Ophthalmol. Vol. 118, pp. 69-77

[2] Ido Perlman (2011). The Electroretinogram: ERG by Ido Perlman. Webvision.

[3] Ovcharenko, S[ergey] \& Minakov, V[italiy] (2017). Application of the Artificial Neural Networks for Diesel Diagnostics, Proceedings of the 28th DAAAM International Symposium, pp.1208-1212, B. Katalinic (Ed.), Published by DAAAM International, ISBN 978-3-902734-11-2, ISSN 1726-9679, Vienna, Austria DOI: 10.2507/28th.daaam.proceedings. 168

[4] Alsulaiman, Mansour \& Muhammad, Ghulam \& A Alomari, Mohammed \& A Alshehri, Mohammed \& Ali, Zulfiqar \& Mahmood, Awais. (2011). An Automatic Diagnostic System for Medically Disordered Voice.

[5] Bodusz, Wojciech \& Miodonska, Zuzanna \& Badura, Pawel. (2018). Approach for spectrogram analysis in detection of selected pronunciation pathologies. 3-11. 10.1007/978-3-319-70063-2_1.

[6] Bolshakov A. A., Eliseev D. V., Musatov V. Yu., Sidorovich O. V., Elizarova S. Yu., Koroleva I. V., Druzhin Yu. I., Sklyarova E. Yu. (2010). Combined method of automated diagnosis of diseases of the urinary system against the background of connective tissue dysplasia - SSTU Bulletin, Vol. № 2c / part 4

[7] Bakutkin V.V., Zaiko Yu.N., Lepesko A.S., Skidanov A.N. (2008). Development of a system for the classification and diagnosis of ophthalmologic diseases based on artificial neural networks - Proceedings of Saratov University. New series. Physics Series, Vol. № 1/part 8. - pp. 36-41.

[8] Al, Mabrouk Mohammad (2011). Hardware-software tools and algorithms for the recognition of heart pathologies based on perceptron networks: diss. Cand. tech. Sciences: - Vladimir, 203 p.

[9] Rebrova, O. Yu. (2003). Mathematical algorithms and expert systems in the differential diagnosis of stroke: diss. Dr. med. Sciences: - Moscow, 325 c.

[10] E.A. Chernyaeva (2008). Algorithmic approaches to the automated diagnosis of acute renal failure based on classification decision-making procedures - VSTU Bulletin, Vol. № 11/part 4

[11] Podvalny S. L., Plotnikov A. V., Okrachkova I. V., Firsov O. V., Verikovsky V. A. (2010). A computerized system for diagnosing blood flow insufficiency based on nonlinear discriminant analysis - VSTU Bulletin, Vol. № 5/part 6

[12] W. Frawley, G. Piatetsky-Shapiro, C. Matheus (1992). Knowledge Discovery in Databases: An Overview. - AI Magazine, pp. 213-228.

[13] D.V. Vershinin (2011). Diagnostics of the current state of complex dynamic objects using the parameters of the simulation model, dis. Cand. tech. Sciences, pp. 132

[14] Hastie T., Tibshirani R., Friedman J. (2001). The Elements of Statistical Learning, Springer, pp. 533 